\title{
La investigación social como actividad institucionalizada y como experiencia socio-histórica
}

\author{
THE SOCIAL RESEARCH AS INSTITUTIONALIZED ACTIVITY AND AS SOCIO-HISTORIC EXPERIENCE
}

Teresa Pacheco-Méndez (kat@unam.mx) Universidad Nacional Autónoma de México (Ciudad de México, México) ORCID: 0000-0003-2498-7113

\begin{abstract}
As any social activity, research in social sciences is institutionalized by the action of individuals, establishing certain mechanisms that regulate -through defined organizational patterns- its social and institutional activities. In this way, the social research developed in higher education institutions is experienced by their actors as an established and objective reality that precedes the actual individual; a reality attached to a clear definition of roles, situations and behaviors. From a sociological perspective, and confronting the interference of the current information and communications technologies, in this paper are explored the obstacles and opportunities facing the institutionalized condition of social research, and their actors in societies with different types of development. For that purpose, we look into the connection of four dimensions of the problem: the expansion and dispersion of human and social sciences; the institution as a regulatory structure for the organization of the research activity; the academic researcher as the main actor, and finally, the role of "academic peer" as an arbiter of a competition more institutional than cognitive.
\end{abstract}

Key words: social sciences, social research, higher education institutions, normative framework, social research actors.

\section{Resumen}

Como toda actividad social, la investigación en el campo de las ciencias sociales se institucionaliza por la acción de los individuos, instaurando ciertos mecanismos que regulan -a través de pautas organizacionales definidas- su quehacer social e institucional. Es así que la investigación social desarrollada en las instituciones de educación superior es experimentada por sus actores como una realidad establecida y objetiva que antecede al individuo actual, una realidad sujeta a una clara definición de roles, situaciones y comportamientos. Desde una perspectiva sociológica, y ante a la injerencia de las actuales tecnologías de la información y la comunicación, en este trabajo se exploran los obstáculos y las posibilidades que enfrenta la condición institucionalizada de la investigación social y la de sus actores en sociedades con distintos tipos de desarrollo. Con tal propósito nos detenemos en el enlace de cuatro dimensiones del problema: la expansión y dispersión de las ciencias humanas y sociales; la institución como marco normativo de la organización de la actividad de investigación; el académico-investigador como principal actor y, por último, la función del "par académico" como árbitro de una competencia más institucional que cognitiva. 
Palabras clave: ciencias sociales, investigación social, instituciones de educación superior, marcos normativos, actores de la investigación social.

\section{Introducción}

El proceso de institucionalización de la investigación ha sido un tema considerado de gran importancia por parte de la historia, la sociología de la ciencia y la sociología de la educación. La historia de la ciencia, arraigada a una visión demarcada por paradigmas, se ha centrado en la recuperación del desarrollo evolutivo del razonamiento y del método científico en las distintas etapas de desarrollo de la sociedad. A diferencia de este acercamiento más de tipo historiográfico, la sociología de la ciencia y del conocimiento, ha dirigido su atención a los factores sociales constitutivos de la ciencia y del conocimiento de lo social, así como al papel social que el científico ha desempeñado como productor de conocimiento.

Situada de manera más cercana a la complejidad de la vida social y sus implicaciones educativas, la sociología de la educación se inclina por el estudio de las instituciones, su dinámica social y sus respectivas formas de organización y administración; la elección de problemas, así como el diseño de estrategias metodológicas están estrechamente vinculadas a las circunstancias del presente, es decir, tanto con las prácticas vigentes dentro de la disciplina, como con la coyuntura institucional, social y política. En esta perspectiva, este trabajo revisa algunas las formas de interacción entre conocimiento social, investigación, actores e institución, a fin de posicionar a la estructura social de la educación en la intersección tradiciónmodernidad.

Se persiguen dos propósitos. Primero, ofrecer una plataforma de análisis y de debate sobre las actuales condiciones de institucionalización y reificación (Berger y Luckmann 1983:116 y 119) de la producción intelectual en ciencias sociales que se lleva a cabo en las instituciones de educación superior en un entorno mediado por recursos tecnológicos. Segundo, identificar la condición institucionalizada de la investigación social y sus actores, recuperando sus principales recursos -incluidos los tecnológicos- para posicionarse más allá de los límites institucionales, y proyectarse como posibilidad de cambio y de impulso para el desarrollo de las ciencias sociales como campo científico.

Para cumplir con tales propósitos, distinguimos cuatro planos de la realidad donde se despliegan relaciones de interacción entre conocimiento social, investigación, institución y actores; son planos interconectados y mediados por el uso de recursos tecnológicos que, en conjunto, dan cuenta del funcionamiento de la actividad intelectual desarrollada por el académico-investigador en las instituciones de educación superior. Tales dimensiones son: la expansión y dispersión de las ciencias sociales como campo científico; la institución como marco normativo de la organización de la actividad de investigación; el académico-investigador como principal actor y, por último, la función del "par académico" como una figura que, desde su muy particular compartimiento cognitivo, detenta una "autoridad intelectual" instituida para juzgar cuándo una aportación al conocimiento es o no relevante.

En el entrecruzamiento de estos planos de la realidad, cobra sentido e inteligibilidad tanto la complejidad de la producción intelectual, como los riesgos en los que se incurre al optar por procedimientos de gestión aislados -entre los que figuran los recursos digitales-, como medios para valorar la relevancia de la producción intelectual. Al desanclar a la investigación social de su contexto de origen, disposiciones tales como políticas educativas y de investigación, quedan destinadas a obstaculizar y distorsionar el sentido de la experiencia intelectual que se lleva a cabo en las instituciones de educación superior, una actividad que ha adoptado nuevas prácticas derivadas del uso de las redes de información y comunicación digitales. A 
pesar de su entrecruzamiento, cada plano atiende a dinámicas e intereses específicos, unos más distantes que otros con respecto a los intereses que persigue la producción de conocimiento; es en razón de ello que aquí los abordamos por separado sin pretender con ello, soslayar sus respectivos anudamientos.

\section{La expansión y dispersión de las ciencias humanas y sociales. Su inaccesibilidad desde los parámetros institucionalizados}

La producción histórica de conocimiento sobre el hombre y el mundo social se compone de un sin número de núcleos de contexto que, a lo largo del tiempo, han dado lugar al establecimiento de tendencias, corrientes y/o escuelas de pensamiento, abarcando desde dimensiones macro sociales hasta procesos de interacción de alcance micro-social. Lejos de pretender establecer sistemas clasificatorios universales, entendemos a la historia de las ciencias sociales como el conjunto de espacios/tiempo -que condicionan y son producto de la interacción del hombre- donde confluyen visiones del mundo, momentos y coyunturas, planos, niveles, estructuras de pensamiento y actores sociales. Es en función de las condiciones a las que históricamente pertenezcan tales espacios/tiempo donde tendrán arraigo los respectivos debates en torno a la "objetividad" del conocimiento alcanzado, así como su respectiva particularidad y especificidad socio-histórica. Al no permanecer al margen de las condiciones que lo producen, el conocimiento de lo social diversificado en dominios disciplinarios, encuentra en la coyuntura que le da origen su potencial explicativo y reflexivo.

El desarrollo de las ciencias sociales se encuentra íntimamente vinculado con la historia de la sociedad, con sus guerras, conflictos y movimientos sociales, pero también con la constante recomposición económica, social y cultural, así como de las fuerzas políticas que, a nivel local y mundial, configuran los distintos escenarios para el reacomodo de la estructura social. Si bien la producción del conocimiento en ciencias sociales está sujeta a la condición histórica de los fenómenos y de los cambios sociales y culturales que son su objeto de estudio, estas ciencias se constituyen como portadoras de un potencial innovador en permanente transformación que se traduce no solo en un intenso debate teórico, mucho más diverso y menos universal que el de las ciencias exactas y naturales, sino también en una constante fragmentaciónhibridación (Dogan y Phare 1993:11) del conocimiento, consecuencia de la progresiva complejidad del mundo social.

Tales efectos del cambio social en el comportamiento de las ciencias sociales, dan lugar a una nueva dinámica en el avance y en conformación del conocimiento sobre el mundo a la que Dogan y Phare definen como "dominios híbridos" (1993:79). Estos se producen como resultado de la intersección de las disciplinas, distinguiendo, por un lado, aquellos dominios formales institucionalizados como subdisciplinas o como unidades o programas ubicados en el cruce de varias disciplinas, y por otro, los dominios informales que se identifican más con la apertura de un campo de estudio. En su opinión, la presencia cada vez mayor de "dominios híbridos", tiende a cuestionar la estructura tradicional de las ciencias sociales (Dogan y Phare 1993:105 y 135). En este permanente reacomodo y reorganización de la producción del conocimiento de lo social, las TIC vienen a incorporarse en un doble sentido: como dispositivo para estimular el surgimiento de "dominios híbridos", y como componente intrínseco que modifica de manera decisiva la realidad y los procesos de interacción social que son objeto de estudio de las ciencias sociales, principalmente de la sociología.

Estimulada por las actuales herramientas electrónicas, la hibridación del conocimiento de lo social puede fomentar la producción de un conocimiento innovador e integrador donde confluyan y se articulen los avances especializados provenientes de los diversos dominios disciplinarios de las ciencias sociales. No 
obstante, también existe la posibilidad de que tal segmentación de conocimiento y que el uso indiscriminado de recursos tecnológicos, no siempre de lugar a un avance innovador, sino más bien a una mayor fragmentación que opere obstaculizando cualquier intento de integración cognitiva, promoviendo la permanencia de una visión a-histórica y estereotipada sobre la realidad social actual.

Esta última dificultad suele ser la experimentada en la práctica institucionalizada de la investigación; esto se presenta cuando el investigador a pesar de contar en la actualidad con una red de acceso e intercomunicación vasta y multi-direccional con su ámbito de especialidad, suele omitir e incluso desconocer consciente o inconscientemente, tanto el núcleo de contenido de su disciplina, como también las innovaciones generadas por los dominios disciplinarios y de especialización diferentes pero complementarios al suyo. En este caso, el efecto de la segmentación suele verse reflejado en el seno de las instituciones educativas donde la actividad de investigación, además de ser considerada desde el punto de vista administrativo como una función "sustantiva" de la institución, también es regulada para su ejecución por un ordenamiento normativo del mismo orden. Es en razón de esta naturaleza institucional que la condición del investigador, la tarea que él desarrolla, y los productos o la productividad que de él se desprendan, disten de ser congruentes tanto con una actividad intelectual de orden científico, como con las posibilidades de uso de los grandes acervos virtuales de información actualmente disponibles.

Los análisis efectuados por Dogan y Phare (1993) y por la Comisión Gulbenkian (Wallerstein 1996) sobre las condiciones y perspectivas del desarrollo de las ciencias sociales y de sus respectivos dominios disciplinarios, han mostrado la co-existencia de diversos marcos interpretativos y de contexto que, dependiendo su inserción socio-histórica y de su particular orientación frente al conocimiento, ponen énfasis en uno o varios rasgos constitutivos del proceso de producción intelectual y científica sobre lo social, así como en algunas de las formas bajo las cuales éstos son asimilados e incorporados por las instituciones educativas y por su respectivas comunidades académicas. Bajo este mismo razonamiento, los actuales canales de comunicación y de intercambio de información y conocimiento, han afectado y modificado procesos sociales y cognitivos en la medida que ya forman parte de la vida cotidiana de los individuos, de los grupos, de las relaciones interpersonales y de la sociedad global. Hoy en día, "el conocimiento y la información son elementos decisivos en todos los modos de desarrollo, ya que el proceso de producción siempre se basa sobre cierto grado de conocimiento y en el procesamiento de la información. Sin embargo, lo que es específico del modo de desarrollo informacional es la acción del conocimiento sobre sí mismo como principal fuente de productividad" (Castells 2005:43).

Esta condición socio-histórica del desarrollo de las ciencias sociales nos plantea tres tareas por resolver: primero, identificar dónde radica el carácter innovador del conocimiento y de la investigación en ciencias sociales en el contexto de la expansión de los recursos tecnológicos; segundo, hasta qué punto las formas de resolución institucionales para la investigación dentro este campo de conocimiento, pueden mantener cierto margen de autonomía con respecto a la sociedad en red, al mundo digitalizado, y a las relaciones de poder social e institucional existentes. Por último, en qué medida la habituación de los individuos a los nuevos patrones de relación y de comunicación global puede darse a un ritmo tal que permita la instauración de nuevos mecanismos de interacción y creatividad antes impensables. El cumplimiento de estas tareas está supeditado a las posibilidades de poder entablar un debate intelectual en el que al menos puedan esclarecerse, por un lado, el significado de un conocimiento "innovador" en un entorno global, digital y multi-referenciado y, por otro, la capacidad de las instituciones educativas y de investigación para adecuarse a esta demanda. 


\section{La institución. Marco normativo de la organización de la investigación social}

Las iniciativas para organizar o reorganizar las estructuras institucionales que dan espacio a la investigación en ciencias sociales han dependido del capital cultural y científico detentado por cada país y por cada institución académica o de investigación; una capacidad que ha determinado una mayor o menor identificación o distancia de esta actividad intelectual con respecto a los criterios del mundo de la ciencia. Tales parámetros de organización se han visto afectados por las actuales condiciones espacio-temporales originadas por la incorporación de nuevos entornos sistémicos de información y comunicación propios del ciberespacio, un entramado donde el habitual funcionamiento institucional se ve rebasado en sus tradicionales límites de espacio y tiempo.

En razón de tales condiciones socio-históricas, la organización institucional de las ciencias sociales se encuentra sujeta a un triple sistema de relaciones. Primero, al ordenamiento social e institucional que da lugar a la socialización y legitimación de representaciones objetivadas (Berger y Luckmann 1983:52, 8183) acerca del quehacer intelectual y científico; segundo, al caracterizado por la gama de procedimientos y convenciones propios de los diversos dominios disciplinarios y especializados que norman y establecen criterios particularizados a los que se acoge la investigación como actividad intelectual socialmente reconocida. Por último, la nueva naturaleza de las prácticas de la investigación social, ahora mediadas por recursos tecnológicos potencialmente abiertos a horizontes cuya dinámica se define precisamente por la transgresión de fronteras cognitivas e institucionales. Producto de esta interconexión resultan representaciones y prácticas institucionales, de grupo y de relación interpersonal sobre el quehacer científico y la producción intelectual, representaciones y prácticas que no siempre coinciden en intereses y propósitos.

Al primer sistema de relaciones, corresponden los tipos de producción intelectual respaldados y asegurados por la norma institucional y su consecuente asignación de papeles y roles; figuran como modalidades reconocidas por un colectivo de académicos cuya experiencia y trayectoria se encuentra determinada más por "un capital de poder sobre el mundo científico, que por un capital de autoridad propiamente científica" (Bourdieu 2003:103). Son normas que al cobrar forma de código cultural socialmente compartido y reconocido sobre la investigación, condicionan la estructura, organización y distribución de los intereses que entran en juego en esta actividad, dirigen los procesos y las prácticas científicas que ahí se realizan, y aseguran su socialización y permanencia. Algunas de estas prácticas son: la distinción de perfiles académicos en función de capacidades y habilidades específicas para desempeñar actividades de investigación; la importancia atribuida al trabajo colectivo sobre el individual y viceversa; el predominio del discurso formal (a-histórico) de la ciencia, del político-institucional, y de aquel forjado por el investigador a lo largo de su formación social, institucional y cognitiva.

En el segundo sistema de relaciones concurren estilos y mecanismos de circulación de la producción intelectual, expresados bajo formas específicas de intercambio de información y/o de comunicación entre quienes recae el quehacer institucional de la investigación. Entre éstos destacan los siguientes: a) la participación en redes académicas, videoconferencias, congresos, simposios, mesas redondas, etcétera, espacios frecuentemente considerados como medios de certificación $\mathrm{y} / \mathrm{o}$ de reconocimiento institucionales de actividades de investigación y de cumplimiento de requisitos de evaluación, incluso para obtención de grados académicos; b) los mecanismos para el otorgamiento de premios, nombramientos y otro tipo de estímulos, de status, de prestigio y económicos, obtenidos una vez cubiertos los requerimientos institucionales y político sociales establecidos; c) los que han validado que la publicación de productos académicos sea reconocida como uno de los principales referentes de medición de la 
productividad y de la innovación científica generada en las instituciones educativas y de investigación y por los actores a ellas adscritos; d) los que consideran válida la figura institucionalizada del "par académico" en su rol de pretender juzgar de manera lineal y unidireccional aquel conocimiento que con frecuencia escapa a los límites de su capacidad cognitiva real.

Por último, en el tercer sistema de relaciones interactúan, por un lado, las tradicionales actividades de investigación habitualmente confinadas al espacio regulado de la institución académica, y por otro, las que pretenden re-significarse haciendo uso de recursos tecnológicos sin lograr desanclarse de las arraigadas practicas instituidas. Un caso es el mencionado por Bustos y Feltrero (2005) cuando la escritura abandona su linealidad para ahora utilizar el hipertexto, una práctica que -y aquí diferimos de los autores- no siempre asegura cambios de perspectiva epistemológica. Otro caso evocado por ellos es precisamente la creación de espacios editoriales virtuales; en ellos sucede algo semejante al adoptar -calcar- de manera prácticamente idéntica, los procesos y la normativa editorial producida por las instituciones tradicionales.

Bajo esta dinámica, el quehacer intelectual en ciencias sociales en las instituciones de educación superior se rigidiza y estandariza, estereotipando los procesos de producción, circulación y consumo de la investigación, aún y a pesar de la incursión de las nuevas tecnologías de la información y la comunicación. Estas prácticas solo favorecen el predominio de un "poder sobre el mundo científico", profundizando las relaciones de desigualdad entre los distintos sectores y actores sociales involucrados en la producción intelectual. Un escenario muy lejano de la posibilidad de recuperar un capital de autoridad propiamente científica, donde "el despliegue de poder para producir la voluntad de saber" (Popkewitz 1994:111) provea a la producción intelectual de un nuevo significado.

Algunas de las prácticas señaladas se reflejan en el mantenimiento de la gama de temas y/o líneas de investigación más concurridos por el investigador, elecciones motivadas por preferencias en ocasiones colectivas y de grupo tales como: las inercias teóricas y metodológicas; las pautas, los estilos y los usos de la investigación institucionalmente reconocidos; modas, tendencias, corrientes y escuelas de pensamiento dominantes, entre otras. Son prácticas tradicionales e incluso digitalizadas que en el espacio institucional son consideradas para su evaluación como indicadores de "relevancia", "rigor" y "pertinencia", muy a pesar de que adolezcan de referentes de contrastación sólidamente diseñados. Esto toca en buena medida a la información y al conocimiento que circula en la red ya que, "a pesar de que la cantidad de información puesta en circulación en Internet es incomparablemente superior a la de otros medios, la acreditación o cualificación de esa información es realmente menor o de peor calidad, en parte porque es preciso idear nuevos mecanismos o generar nuevas instituciones que desempeñen esas funciones en el entorno digital" (Bustos y Feltrero 2005:4).

Un componente esencial de este entorno institucional donde se desarrolla la investigación social es el papel desempeñado por las políticas públicas. El alcance de su acción mediadora entre, por un lado, la esfera política y económica y, por otro, las directrices de desarrollo de las instituciones de educación superior, difiere en función del tipo de sociedad que se trate. En economías desarrolladas el peso de políticas públicas en la economía, la educación y el desarrollo científico y tecnológico, afecta directamente a la estructura y funcionamiento de las instituciones, incluidas las de investigación y de educación superior -trascendiendo incluso sus marcos organizativos. En cambio, para las economías en desarrollo la incidencia de tales políticas es marginal en cuanto a su impacto en el ámbito económico y de desarrollo científico y tecnológico, restringiendo su alcance y penetración en lo educativo. En este último caso, los efectos de las políticas públicas en la estructura educativa se limitan a la instauración de medidas regulatorias de recursos financieros, humanos y tecnológicos que, sin trascender ni modificar la estructura y 
funcionamiento de las instituciones, atienden de manera circunstancial y aleatoria, aspectos de distribución y gestión de recursos físicos, humanos y financieros.

Iniciativas que en el caso de América Latina son instrumentadas y administradas por dependencias gubernamentales para el fomento de la educación, la ciencia y la tecnología, así como por las propias instituciones de educación superior; estas últimas a través de la oferta de diversos programas de evaluación para la asignación de estímulos económicos complementarios al salario, así como otros destinados al otorgamiento de recursos adicionales para proyectos de investigación y docencia. Todas ellas, acciones cuyos parámetros son más afines con las pautas de racionalización administrativa, o en su caso, tendientes a formalizar orientaciones temáticas a partir de problemas sociales considerados coyunturalmente prioritarios. Con ello nos referimos a lo que Bourdieu denomina "habitus", una categoría por él construida y desarrollada a lo largo en sus trabajos sobre el "campo social". Tal como la define, el "habitus" es un "sistema de disposiciones orientadas a la práctica, constituyéndose como el fundamento objetivo de conductas regulares y, por lo mismo, de la regularidad de las conductas... podemos prever las prácticas ... precisamente porque el habitus es aquello que hace que los agentes dotados del mismo se comporten de cierta manera en ciertas circunstancias" (Bourdieu 1986:40).

Es así que la investigación social considerada como función "sustantiva" de las instituciones de educación superior se reifica, asimilando y haciendo propias aquéllas disposiciones fijas y duraderas que históricamente la han consolidado como una actividad institucionalizada con los rasgos que aquí brevemente hemos descrito. Esta condición le ha reducido significativamente su margen de acción -sin anularlo- para revertir esta tendencia y proyectarse, con el respaldo de las TIC, como posibilidad de avance e innovación en el terreno del conocimiento.

Si bien ha sido en razón de los cambios producidos en la base de la organización de la sociedad que las ciencias sociales se han transformado y adecuado -en el seno de las instituciones educativas y de investigación- a las nuevas demandas, la lectura que se haga sobre su trayectoria y desarrollo dará cuenta, ya sea de las posibilidades de apertura de pensamiento y de interpretación que puedan proporcionar sobre una realidad en constante movimiento y cambio, o por el contrario, de las limitaciones derivadas de su inserción al espacio de las instituciones de educación superior. Es en esta dinámica constitutiva del pensamiento social donde se sitúa el presente y futuro del papel desempeñado por su principal actor: el investigador.

\section{El académico-investigador}

El investigador como actor social e institucional es producto de un doble proceso, uno de formación y otro de socialización. De formación, como sujeto constituido bajo la influencia de un contexto histórico-social y de unas instituciones culturales que inciden de manera decisiva en la idea que él se forje sobre el mundo social con el que se relaciona e identifica. De socialización, como investigador instruido por el discurso formal de la ciencia y entrenado "en una modalidad de visualizar y pensar lo social" (Andrade 2010:162). Como resultado de esta doble formación, el investigador elabora en distintos momentos de su trayectoria, lecturas o representaciones sobre cómo él construye la realidad del mundo social; estas lecturas cobran materialidad en los procesos de investigación hoy mediados por la institucionalización de las nuevas prácticas tecnológicas, una herramienta que, si bien abre un horizonte de posibilidades, también lo hace de dificultades. 
Para autores clásicos como Barnes (1985:63), la importancia de tales representaciones estriba en su facultad para condensar tanto valores sobre el conocimiento, como procesos de incorporación y actualización de la cultura a la que pertenecen los actores que las producen. Ya en su época, Barnes distinguía representaciones que el investigador asume de manera rutinaria y de acuerdo a como mejor convenga en el plano instrumental, pero también aquellas otras que operan más como un recurso cultural para la generación de nuevos conocimientos. Las primeras -representaciones asumidas por el investigador de manera rutinaria- atienden preferentemente a los usos y costumbres establecidos, así como al reconocimiento institucionalizado por parte de grupos y organizaciones. Son representaciones socialmente útiles que se imponen a través de diversos mecanismos como:

a) La repetición, en cuanto a los pasos a seguir para la elaboración de protocolos de investigación en los que sean debidamente retomadas las exigencias de contenido consensuadas (antecedentes, justificación, problema, objetivos, marco teórico, cronograma y metas). Requerimientos formales en los que con frecuencia se confunde: la identificación de un problema con la descripción de un objeto; la construcción de categorías con la selección de teorías y conceptos como contenidos cerrados; el uso de recursos técnicos como indicadores de algo que se quiere conocer con la idea de que lo arrojado por el indicador dará cuenta de lo que se quiere conocer (Zemelman 2005).

b) Alinearse a la norma institucional, es decir, dictar conferencias en foros internacionales; publicar artículos, capítulos de libro y otros, dirigir tesis, y otros requisitos contemplados por la normatividad, de acuerdo con el nivel y categoría de una plaza ocupada en una institución de investigación o de educación superior.

c) Imitación e inercia como el efectuar elecciones temáticas, teóricas y metodológicas de acuerdo con las preferencias dominantes en el grupo social o institucional de pertenencia. Esto incluye los temas en boga en otros contextos no locales-nacionales.

Acciones todas ellas caracterizadas por el despliegue de una economía de esfuerzo motivada con frecuencia por la búsqueda de legitimación y el mantenimiento de ciertas posiciones sociales e institucionales alcanzadas, es decir: ser investigador reconocido, ser emérito, o detentar una categoría y nivel altos dentro de la institución; defender otros prestigios adquiridos como "ser de la escuela de ' $X$ ' autor", "ser constructivista", o en su caso, ser reconocido por el grupo institucional de pertenencia. Todas ellas son representaciones sobre una actividad intelectual altamente estereotipada donde las prácticas instituidas se reafirman, sin que medie un esfuerzo crítico y reflexivo que logre modificarlas y revitalizarlas. Son representaciones que en la actualidad incorporan a los recursos tecnológicos como meros accesorios que facilitan el quehacer artesanal de la investigación, sin modificar miradas, propósitos y contenidos ya aprendidos.

El segundo tipo de representaciones distinguidas por Barnes -y entendidas como recursos culturales para la generación de nuevos conocimientos- se presentan como variantes ocasionales, resultantes siempre de condiciones y de recursos culturales anteriores. Son representaciones construidas a partir de una experiencia individualizada -mas no individualista- vinculada más con la realidad histórico-social que con el sentido común y la opinión generalizada. Una experiencia donde las prácticas se renuevan constantemente gracias a un esfuerzo reflexivo y crítico sobre un entorno siempre cambiante y resignificable. Algo cercano a lo propuesto por Andrade en el sentido de que "habría que hablar en vez de mundo social sin más calificativos, de sujetos que habitan un mundo que ellos mismos contribuyen a construir" (2010:155) de manera no siempre consciente ni reflexiva. Incluso el incipiente uso que el 
investigador hace de los actuales recursos tecnológicos puede llegar a representase no solo en términos de acceso a la información y conocimientos disponibles, sino en la posibilidad de instaurar mecanismos dinámicos de interacción entre individuos y grupos donde se ponga en juego su competencia epistémica para seleccionar, depurar y producir conocimiento.

De no tomar conciencia de la importancia que tiene el fomentar este segundo tipo de representaciones, cobrarán mayor fuerza los efectos de las estructuras cognitivas institucionalizadas, llegando a "ejercer dirección sobre las prácticas sin que puedan ser rediscutidos por el sujeto" (Andrade 2010:155). El predominio de las primeras representaciones sobre las segundas hace entonces que el buen académicoinvestigador sea considerado como aquel que, en términos de Bourdieu, ha entendido bien las reglas de un juego "científico" objetivado e institucionalizado. O bien, como lo describe Zemelman al señalar que quien no se atreve a tomar distancia crítica de su posición con respecto al conocimiento, a la realidad social y a la institución, no podrá "construir conocimiento; quien busque mantenerse en su identidad, en su sosiego y en su quietud, construirá discursos ideológicos, pero no conocimiento; armará discursos que lo reafirmen en sus prejuicios y estereotipos, en lo rutinario, y en lo que cree verdadero, sin cuestionarlo" (2005:72).

Cobijada por los preceptos de las políticas públicas dictadas en materia de educación, ciencia y cultura, la práctica del investigador se adapta a los marcos para él creados y a los intereses a los que el discurso y la retórica institucional lo comprometen. Se trata de "políticas individualistas e individualizantes que han premiado por mérito a quien ha sabido mejor ajustarse a las reglas de la academia, [y] han desmembrado la concepción colectiva de la creación de conocimiento" (Naidorf 2013:6). Aún con los recursos desplegados por la tecnología actual, las prácticas del investigador quedan supeditadas a su capacidad y voluntad para hacer frente a brechas culturales y epistemológicas que interfieren en la posibilidad de revertir los modos instituidos de acceder al conocimiento de la realidad social. Brechas que no solo afectan al investigador, sino también a las condiciones bajo las cuales es puesta en circulación -en la red- la información y el conocimiento. "La entropía informacional presenta múltiples características como lo son el desorden, la redundancia, la esterilidad, la inautenticidad de la información, etc." (Bustos y Feltrero 2005:15).

Es así que la objetividad construida socialmente en torno al mundo de las ciencias sociales ha llegado a convertirse en su contexto de referencia, en un producto social que depende de los presupuestos aceptados y regulados por la norma institucional y en especial, con la manera legítima de regular el eventual conflicto. En el sentido planteado por Berger y Luckmann, la objetivación del mundo institucional, en este caso de la investigación en ciencias sociales "por masiva que pueda parecerle al individuo, es una objetividad de producción y construcción humanas donde siempre existirá ... en la conciencia la posibilidad de cambiarlas o abolirlas" (1983:81-83).

\section{El "par académico": árbitro de una competencia institucional o cognitiva}

La "revisión de pares" es la práctica que detenta uno de los reconocimientos social e institucionalmente más extendidos y fortalecidos. Cuenta con el respaldo y legitimación de comunidades científicas, de sectores sociales pertenecientes a la alta política, de instituciones, y de grupos e individuos vinculados formal e informalmente con las actividades desempeñadas en las instituciones de educación, ciencia, cultura y desarrollo tecnológico. Muy a pesar de las ilimitadas posibilidades puestas a disposición en la red para: acceder al conocimiento, para interaccionar entre autores, "pares" y usuarios; para establecer una dinámica de interlocución entre especialistas, y para construir nuevos mecanismos que permitan evaluar 
la relevancia de un producto intelectual, el sistema de "revisión de pares" permanece prácticamente sin cambios.

La función de la "revisión de pares" ha sido concebida en términos institucionales ampliamente aceptados como un proceso en el que se decide cuándo un producto intelectual merece ser reconocido socialmente como novedoso, pertinente y riguroso "científicamente". Es una función que abarca no solo la valoración para determinar si un producto intelectual puede o no ser reconocido, premiado, divulgado y/o publicado a través de algún mecanismo de distinción pública y de difusión impresa o digital (libro, revista, anuario, etc.), sino que también es considerado como el "filtro" para dictaminar sobre el otorgamiento de emolumentos a través de premios, convocatorias, nombramientos y de posiciones laborales.

En ambos casos, el énfasis en el cumplimiento de los requisitos formales a ser cubiertos está claramente establecido, asumido y consensuado por los actores involucrados (editores, comités, jurados, pares y autores), ya que se trata de aspectos empíricos debidamente instituidos, aunque arbitrariamente desarraigados de la especificidad cognitiva del contenido del que tales productos a valorar son portadores. En la investigación social, el reconocimiento que los distintos actores involucrados hagan de la problemática cognitiva y epistemológica involucrada en la manufactura del producto a "revisar", "evaluar" y "juzgar", variará respectivamente en relación directa con aspectos tales como: sus antecedentes de formación, la función institucional desempeñada por cada uno de ellos, la particularidad de su experiencia en el campo de conocimiento de lo social, y por último, su contacto con el desarrollo e hibridación de aquellos ámbitos de especialidad provenientes de otros dominios disciplinarios vinculados al contenido del producto académico sujeto al proceso de "revisión de pares". Esto da cuenta de cómo cada uno de los actores involucrados en la "revisión de pares" es poseedor de capacidades, experiencias y capitales culturales altamente diferenciados, no solo entre ellos, sino también en relación con el producto académico con el cual cada uno establece una relación y una acción distinta, difícilmente de consenso.

Los resultados arrojados por la experiencia de la "revisión de pares" han dado lugar a controversias y desencuentros que ponen en entredicho la eficacia de tal procedimiento. En este debate se han esgrimido argumentos que oscilan a favor y en contra, ya sea de la virtud o de la perversión promovidas por dicha práctica. La oposición generalizada entre el "par" (elegido por criterios y entidades constituidas por disposiciones y normas institucionales) y el responsable o autor del producto académico a juzgar, ha contribuido a una progresiva pérdida de credibilidad en estos procesos. A ello se suma el limitado uso que la evaluación entre "pares" hace de los bancos de información disponibles en la red, a fin de identificar de manera amplia y a la vez particularizada, aquéllos trabajos situados en el ámbito de especialidad, y en las zonas de intersección o hibridación afines al producto evaluado -y considerarlos como referentes complementarios para dar sustento al juicio emitido por el "par".

Los acuerdos a favor de la relevancia de la función del "par" han sido reiteradamente expresados por autoridades y funcionarios institucionales, por investigadores, y sobre todo por entidades pertenecientes al mercado editorial impreso y digital; pero no menos contundentes son aquellas manifestaciones que con firmeza cuestionan categóricamente la arbitrariedad y ambigüedad sobre la que se continúa llevando a cabo la tarea del "par" y la indiferencia mostrada ante la pobreza de sus resultados.

En el primer caso se asegura que el juicio de pares consiste en la puesta en marcha de un "método ... fundamentado en la concepción de que los más aptos para justipreciar el mérito de un científico son quienes comparten con él el esfuerzo de creación de conocimiento" (Editorial 1996:7). En este caso se hace referencia a que: "El buen funcionamiento del juicio de los pares requiere que los evaluadores no 
actúen influidos por sus intereses personales, por su ideología política o por las presiones de los evaluados. Debe formularse de manera independiente de las instancias administrativas y políticas de la ciencia, pero no invadir los temas que competen a estas, las que, en contrapartida, tienen que aceptarlo, sin contradecirlo o distorsionarlo" (Editorial Ciencia Hoy 1996:7).

En el segundo caso, el argumento esgrimido asegura que: "Las deficiencias del juicio de los pares conducen a injusticias para con las personas y tienden a consolidar desigualdades del desarrollo relativo de disciplinas o regiones, así como a promover una investigación rutinaria y repetitiva, todo lo cual afecta negativamente al quehacer científico" (Editorial Ciencia Hoy 1996:7).

A pesar de su intrínseca inclinación personal, institucional y cognitiva, al "par académico" se le han atribuido cualidades que lo dotan de una imagen que lo libera en automático de cualquier compromiso particular; se le designan funciones institucionales que poco coinciden con las condiciones particulares de su origen y formación social, institucional y cognitiva. En realidad, el designado "par académico" es solo un integrante más de ese conglomerado institucional caracterizado por una enorme heterogeneidad de intereses, compromisos y creencias que en conjunto, mediatizan y condicionan su mirada y su capacidad para distinguir, o al menos aproximarse, al horizonte de conocimiento sobre el mundo social.

En su estado actual, la "revisión de pares" ha dejado de representar un "filtro" confiable en lo que respecta a la calidad y pertinencia cognitiva de un producto académico y de investigación. Si alguna función ha cumplido a cabalidad la preeminencia de la figura del "par", es mantener impoluto el estatus y la jerarquía detentados por las instancias y los medios instituidos encargados de otorgar los respectivos nombramientos, premios, distinciones, o bien de publicar los productos académicos sometidos a su arbitraje. Durante las últimas décadas su función social e institucional se ha consolidado, limitándose a administrar y dosificar la creciente oferta cuantitativa de productos académicos y de investigación; ésta última, un fenómeno masivo ocasionado por las políticas de "evaluación académica" destinadas a la dotación de estímulos económicos que, al pretender atender un problema de compensación salarial, han rutinizado, rigidizado y estandarizado la actividad de investigación desarrollada en las instituciones de educación superior.

Hoy en día, un producto académico de mediana calidad cognitiva puede llegar a ser difundido o reconocido con el respaldo y la garantía ofrecida por la "revisión de pares"; aunque en el caso opuesto encontremos también que un producto académico, situado en la intersección de distintos dominios híbridos de diversa procedencia disciplinaria, quede excluido de la posibilidad de ser difundido, publicado y reconocido por alguno de los medios o instancias social e institucionalmente establecidas. En este sentido, no todo producto académico que supere el "filtro" de la "revisión de pares" es portador de un conocimiento innovador; y viceversa, no toda aportación vetada por el juicio de "pares" pierde relevancia como aporte para el avance del conocimiento de lo social.

A esta problemática no resuelta sobre el verdadero papel jugado por la "revisión de pares" se suma, en el caso de la difusión escrita del trabajo académico, la alta dispersión existente en cuanto la creciente oferta de medios de difusión de la producción académica. Un fenómeno que, favorecido por las posibilidades de la red, ha estimulado el acceso al conocimiento, pero también ha actuado en desmedro del valor epistémico de la producción intelectual, al atomizar y disgregar tanto a grupos de científicos como a "pares" como resultado de una excesiva especialización-fragmentación. En este sentido, Bustos y Feltrero aseguran que: "Cada vez hay más publicaciones y cada vez son más específicas, de manera que solo unos pocos expertos de campos muy determinados intervienen en las labores de revisión y contrastación. Si 
bien este movimiento facilita la selección de textos relevantes, en función de la temática específica de cada publicación, no es menos cierto que reducen las tareas de contrastación y revisión a un reducido grupo de investigadores que, generalmente, se asocian en función de una temática que comparten y en la que están de acuerdo" (2005:9).

El crecimiento y predominio de revistas con un perfil profesional e instrumental, sobre otras más preocupadas por el debate epistemológico y teórico, ha tenido efectos directos sobre las prácticas de investigación social; esto se refleja en la inclinación por aquéllas modalidades y metodologías de trabajo académico donde el dato empírico se sitúa en el centro de atención por parte del investigador. En este tipo de revistas predomina una heterogeneidad y asimetría temática, formativa y disciplinaria entre sus autores, a diferencia de aquéllas revistas cuyo interés por la reflexión teórica y epistemológica favorece su consolidación dentro su ámbito de especialización, reuniendo contribuciones académicas más afines entre sí.

En las últimas dos décadas, la industria editorial impresa y en línea -de orientación profesionalizante e instrumental- se ha expandido considerablemente como resultado del alza en la demanda por publicar. Aun cuando su capacidad para dar respuesta a tal demanda ha obedecido a pautas de eficiencia ajenas a la dinámica y ritmo de la producción de conocimiento sobre la realidad social, ha sido esta industria la única instancia capaz de incorporarse y adecuarse a los mecanismos institucionales, a los criterios académicos y a las prácticas propias del trabajo intelectual, reforzando la inamovilidad de las pautas y normas del trabajo académico en las instituciones de educación superior.

\section{Reflexiones finales}

La actual organización y estructuración para el ejercicio de la actividad de investigación e incluso de la formación de profesionales en ciencias sociales prevaleciente en las instituciones educativas, dista de atender al primado epistemológico de estas ciencias, es decir, a la capacidad de comprensión que de ellas se exige y se espera. Si bien la condición y movimiento de estas ciencias nunca han correspondido con el espacio fijo y estructurado del mundo institucional, concierne a la actividad intelectual y al compromiso de académicos-investigadores el contribuir a desanclar las actuales prácticas instituidas, a fin de apostar por una refundación de su sentido histórico-social a través de la investigación.

Sin duda, este reto debe pensarse desde las condiciones actuales de acceso a la información y de producción de conocimiento en la sociedad en red; condiciones que afectan tanto a la naturaleza del objeto de estudio de las ciencias sociales como al proceso para acceder a su conocimiento. En el mundo digital que vivimos es necesario tener presente, como lo señala Albero y Thibault (2009:57), que el fenómeno técnico es constitutivo del ser humano y de su actividad, de ahí que en ciencias humanas y sociales sea indispensable la construcción de un marco teórico y metodológico capaz de analizar el entrelazamiento entre técnica, prácticas sociales y producción de conocimiento.

Reivindicar la autonomía científica -asegura Bourdieu-, significaría para una comunidad desarrollar la capacidad necesaria para sustraerse de los efectos de dominación que, en el caso de las ciencias sociales, influyen distorsionado el sentido de la competencia científica, haciendo prevalecer dentro de este ámbito del conocimiento una mezcla de intereses ideológicos, pragmáticos y de dominación. Intereses que se traducen en la imposición de definiciones a-priori no solo del capital teórico y epistemológico disponible, sino también de una visión objetivada de los problemas sociales (2003:83-99) y de los tradicionales y nuevos procedimientos para conocerlos, validarlos y acreditarlos, política, institucional y socialmente. 
En lugar de procurar la producción de un conocimiento crítico y reflexivo abierto sobre la cambiante realidad social, la institución, las políticas públicas, las disposiciones a las que atiende el investigador y los medios de circulación de los productos generados por la investigación, se han inclinado a "aislar en guetos a las comunidades científicas para que entre ellos discutan, se evalúen y compitan por obtener premios" (Hernández 2011:16). Esto sin duda alguna ha afianzado el predominio de un pensamiento instrumental más de tipo ideológico que consolida las prácticas instituidas, sobre otro de carácter reflexivo, donde es el cambio cultural el punto de referencia que redefine el sentido de futuro para la investigación en ciencias sociales y donde el papel del sujeto reaparece en escena en toda su complejidad: "Quizá uno de los grandes desafíos que nos hereda el siglo XX es precisamente la complejidad de los sujetos que construyen la historia, que están detrás de los fenómenos que queremos estudiar y que son complejísimos; sujetos múltiples que tienen distintas características, variados espacios, tiempos diversos, y visiones diferentes del futuro desde las cuales construyen sus realidades" (Zemelman 2005:78).

El reto al que hoy nos enfrentamos es complejo y afrontarlo supone una triple tarea: a) reflexionar sobre los alcances de la investigación social institucionalizada como realidad objetiva, b) reconsiderar el potencial de cambio de la dimensión tecnológica asimilada a la condición social de los individuos y al quehacer científico y, por último y de mayor importancia, c) remodelar el espacio institucional a modo de dar cabida a una práctica científica donde la formación social y cognitiva del investigador esté respaldada por el potencial del contenido científico-cultural de las ciencias sociales y estimulada por la dinámica de la especialización-hibridación producto de los cambios sociales.

La visión y la interpretación de la realidad social que el investigador social elabora de manera cotidiana, ajustándolas al esquema editorial vigente, representan formas particulares de objetivación del mundo social de las que escrupulosamente habrá que desentrañar, entre otros: las presiones externas institucionalizadas de las tensiones propias del debate teórico y epistemológico ahí contenidas; los efectos de la dominación de un capital de poder sobre el mundo científico, y por último, la "explicitación de los esquemas prácticos que han construido el principio de elecciones decisivas como la elección de una disciplina, de una especialidad ... o de una revista" (Bourdieu 2003:83).

En esta perspectiva habrá de restituírsele a la publicación su condición de referente para valorar los resultados de la investigación social; habrá que definirla entonces como portadora, no del manejo y destreza para cumplir con requisitos formales e institucionalizados por el mercado editorial, sino más bien como una herramienta para recuperar la tradición científica e innovadora de las ciencias sociales, gracias a "un gran dominio de los recursos colectivos acumulados, y que, a partir de ahí, conserva necesariamente lo que supera" (Bourdieu 2003:37). Un requisito que el "par académico" deberá cumplir a cabalidad para estar en condiciones de emitir responsablemente un juicio convincente y de un incuestionable peso académico.

En países desarrollados y en desarrollo, la capacidad de las ciencias humanas y sociales para propiciar un cambio cultural consecuente con el cambio tecnológico, no depende de la voluntad de aparatos de gobierno o de políticas locales, regionales o globales; radica más bien en la voluntad social para remover los límites de acción que las instituciones y los individuos históricamente se han fijado en cuanto a sus formas lineales y unidireccionales de relacionarse con el entorno. El fin no es tecnologizar toda la actividad desarrollada por el hombre en sociedad, sino que sea con fundamento en la hibridación alcanzada por el pensamiento humanístico y social que la tecnología logre poner a disposición del hombre y de la sociedad aquellas herramientas de razonamiento que le permitan comprender con una mirada renovada la 
complejidad intrínseca del mundo actual, dotándolo por este camino de elementos para efectuar las elecciones de sentido que lo conduzcan a trazar escenarios de futuros posibles.

\section{Bibliografía}

Albero, B. y Thibault, F. 2009. La recherche française en sciences humaines et sociales sur les technologies en éducation. Revue Française de Pédagogie 169: 53-66.

https://www.cairn.info/resume.php?ID_ARTICLE=RFPED_169_0053

Andrade, L. 2010. Revisitando el oficio de sociólogo: notas sobre el habitus de investigador social. Cinta moebio 39: 153-169. doi: 10.4067/S0717-554X2010000300003

Barnes, B. 1985. El problema del conocimiento, pp.53-99. En: L. Olivé. La explicación social del conocimiento. México: UNAM.

Bustos, E. y Feltrero, R. 2005. Internet y el acceso al conocimiento: la articulación de lo epistemológico y lo moral. Madrid: UNED. http://www.uned.es/dpto_log/ebustos/docs/InternetAcceso.pdf

Berger, P. y Luckmann, T. 1983. La construcción social de la realidad. Buenos Aires: Amorrortu.

Bourdieu, P. 1986. Habitus, code et codification. Actes de la recherche en sciences sociales 64(1): 4044.doi: 10.3406/arss.1986.2335

Bourdieu, P. 2003. El oficio de científico. Barcelona: Anagrama.

Castells, M. 2005. La era de la información. Economía, sociedad y cultura. Vol. I. México: Siglo XXI.

Dogan, M. y Phare, R. 1993. Las nuevas ciencias sociales. México: Grijalbo.

Editorial Ciencia Hoy. 1996. El juicio de los pares. Ciencia Hoy 6(33): 7-8.

http://www.cienciahoy.org.ar/ch/hoy33/edit01.htm

Hernández, M.J. 2011. El conocimiento social y el compromiso del investigador en el mundo contemporáneo. Intersticios Sociales 1: 1-19. http://www.redalyc.org/articulo.oa?id=421739489002

Naidorf, J. 2013. Desafíos de diferenciación de la universidad latinoamericana. Sinéctica 40: 1-9. http://ref.scielo.org/9p7tjx

Popkewitz, T. 1994. Política, conocimiento y poder: algunas cuestiones para el estudio de las reformas educativas. Revista de la Educación 305: 103-137.

Wallerstein, I. 1996. Ouvrir les sciences sociales. Paris: Descartes and Cie.

Zemelman, H. 2005. Voluntad de conocer. El sujeto y su pensamiento en el paradigma crítico. Barcelona: Anthropos.

Recibido el 14 Oct 2016

Aceptado el 2 Dic 2016 